\title{
Archéologie, forêt et Lidar : une recherche qui a du relief ! Introduction
}

\section{Archaeology, forest and Lidar: a research which has relief! Introduction}

\author{
Laurent Costa ${ }^{1}$, Laure Laüt ${ }^{2}$, Christophe Petit ${ }^{3}$ \\ ${ }^{1}$ Ingénieur de recherche CNRS, UMR 7041 UMR 7041 ArScAn - Archéologies et Sciences de l'Antiquité, Équipe \\ Monde Grec Archaïque / programme ArcheoFab, Consortium Huma-num Paris Time Machine (Laurent.costa@cnrs.fr) \\ ${ }^{2}$ maître de conférences, Université Paris 1 Panthéon-Sorbonne, UMR 8546 AOROC-Achéologie et philologie d'Orient \\ et d'Occident (CNRS-PSL) (laure.laut-taccoen@univ-paris1.fr) \\ ${ }^{3}$ Professeur à l'Université Paris 1 Panthéon-Sorbonne, UMR 7041 ArScAn Equipe Archéologies environnementales \\ (christophe.petit@univ-paris1.fr)
}

RÉSUMÉ. La technique du LiDAR (Light Detection and Ranging) permet une restitution très fine de la topographie à partir d'un scanner laser embarqué dans un avion. Depuis quelques années, cette technologie rend visible des vestiges archéologiques sur des kilomètres carrés de zones forestières jusqu'alors muettes vues du ciel. Les sites archéologiques sont reconstitués avec leur environnement (routes, parcelles, structures agraires etc.) à des échelles difficilement analysables jusqu'alors. Ce numéro spécial nous offre l'occasion d'un tour d'horizon des approches françaises en matière d'exploitation archéologique du LiDAR sans prétendre, loin de là, à l'exhaustivité, tant les travaux sont foisonnants, en France comme à l'étranger. Mais l'éventail des articles ici réunis reflète la diversité des recherches actuelles, quant aux échelles de travail (site, massifs forestiers, région...), aux procédés d'acquisition LiDAR (avion, drone), aux terrains d'enquête (de l'Aisne à l'Allier, de la Vendée aux Vosges et jusqu'au Mexique) ou aux périodes et fourchettes chronologiques abordées (du temps long de l'histoire agraire au temps court de la bataille de Verdun). Ce sont aussi d'assez nombreux points de méthodes qui sont abordés au fil des articles, concernant le traitement des données LiDAR, l'interprétation manuelle ou semi-automatique des reliefs, les protocoles de vérification sur le terrain, la confrontation avec les données environnementales, etc.

Autant d'éclairages qui, nous l'espérons, viendront témoigner de cette recherche pleine de relief (à tous les sens du terme...) et nourrir la réflexion sur les apports du LiDAR à l'enquête archéologique en forêt, sur les approches à développer pour bien l'exploiter, en travaillant la donnée numérique, mais aussi et toujours l'information historique et la réalité du terrain.

ABSTRACT. The LiDAR (Light Detection and Ranging) technique allows a very fine restitution of the topography from a laser scanner on board an aircraft. For several years now, this technology has been making archaeological remains visible over square kilometers of forest areas that were previously invisible from the sky. The archaeological sites are reconstructed with their environment (roads, plots of land, agrarian structures, etc.) at scales that were previously difficult to analyze. This special issue provides us with an overview of French approaches to the archaeological exploitation of LiDAR without claiming, far from it, to be exhaustive, as there is so much work to be done, both in France and abroad. However, the range of articles gathered here reflects the diversity of current research, in terms of scales of work (site, forest massifs, region, etc.), LiDAR acquisition procedures (aircraft, drone), the fields of investigation (from the Aisne to the Allier, from the Vendee to the Vosges and as far as Mexico) or the periods and chronological ranges covered (from the long period of agrarian history to the short period of the Battle of Verdun). A number of methodological points are also covered in the course of the articles, concerning the processing of LiDAR data, the manual or semi-automatic interpretation of relief, field verification protocols, comparison with environmental data, etc.

We hope that these insights will bear witness to this research which is full of relief (in every sense of the word) and will provide food for thought on the contributions of LiDAR to archaeological investigation in the forest, on the approaches to be developed to make good use of it, by working with digital data, but also, and always, with historical information and the reality of the field.

MOTS-CLÉS. LiDAR, Forêt, Agro-pastoralisme, Archéologie, Parcellaire, Paysage.

KEYWORDS. LiDAR, Forest, Agro-pastoralism, Archaeology, Landscape.

Le LiDAR, acronyme de light detection and ranging, vient s'insérer dans un paysage de la recherche archéologique en pleine mutation technologique. Cette méthode de télédétection 
permettant de scanner le sursol et le sol à l'aide d'un capteur haute densité a profondément renouvelé notre appréhension des vestiges archéologiques. Bien qu'inventée dans les années 1970 (Weishampel 2012), cette technique ne s'est démocratisée que depuis une quinzaine d'années. Grâce à la diminution des coûts de mise en œuvre et à la possibilité d'accès à des outils de traitement de mieux en mieux maîtrisés, l'utilisation de ces données s'accroît massivement dans nos approches archéologiques, provoquant une véritable rupture méthodologique.

En effet, le LiDAR permet de s'affranchir d'une partie des contraintes et limites classiques inhérentes aux méthodes de prospection. Couverture de vastes espaces, haute densité d'information, haute résolution dans les mesures, objectivation des relevés ne sont que les avantages les plus évidents de ces données. Le milieu forestier, particulièrement complexe à appréhender avec les méthodes traditionnelles, en est un terrain d'application privilégié et les exemples d'usage en archéologie sont maintenant nombreux, certains étant devenus des classiques. Citons l'étude, à partir de 2003, des champs bombés conservés en forêt à Rastatt, en pays de Bade (Sittler et Hauger 2007), réalisée par une équipe de l'institut de gestion des paysages de l'université de Fribourg-en-Brisgau. Citons aussi chez nos voisins anglais, l'enquête sur les massifs forestiers de la forêt de Dean en Grande-Bretagne (Devereux et al. 2008) ou plus à l'est, celle de nos collègues autrichiens sur la montagne de la Leitha en Autriche (Doneus 2013), et dans le nord de l'Europe, les recherches menées à Elverum en Norvège (Risbøl et al. 2006). De l'autre côté de l'Atlantique, on peut évoquer les recherches menées dans le Michigan aux États-Unis (Gallagher et Josephs 2008), en Mésoamérique (Canuto et al. 2018 ; Forest et al. 2020) avec des cas d'usage toujours plus nombreux, ou encore dans la forêt tropicale de Guyane française (Mestre et al. 2008). En métropole, c'est la Bourgogne qui fut pionnière, avec le site de Saint-Martin-du-Mont en Côte-d'Or (Beck 2009), où s'est déroulée l'une des toutes premières expériences d'utilisation du LiDAR en forêt en 2006, suivie en 2007 par les investigations en forêt de Haye en Lorraine (Georges-Leroy 2009; Georges-Leroy et al. 2011). Vinrent aussi des levers réalisés dans le Morvan à Bibracte (Bibracte, coll. 2010), dans le Châtillonnais (Goguey et al. 2010, 2018), à Alésia (Vidal 2016), à Autun (Saligny et Mémier 2014), dans les forêts de Fontainebleau (David 2018), de Montmorency (David et Trouvé 2015), de Compiègne (Rassat et Hugonnier 2017), de Bercé (Le Jeune et al. 2017) ou encore du Val-Suzon (Landois, Garcia, Quiquerez 2019)... Cette liste, loin d'être exhaustive, illustre l'utilisation croissante de cette technique dans toutes les régions de France, pour répondre à des problématiques archéologiques variées.

De manière inédite, ces approches ont permis de faire «sortir du bois » des objets linéaires complexes à percevoir comme les structures agraires, les réseaux parcellaires et viaires, remarquablement bien conservés sous forêts et très bien révélés par le LiDAR. Ce fait a été mis en évidence dès les premières utilisations de la méthode en 2000 (Brian, Motkin et al. 2002) et s'est confirmé de façon quasi systématique au fil des nombreuses interventions du même type qui ont suivi dans différentes zones boisées. Le sujet est donc au cœur de notre actualité scientifique et nécessitait qu'on s'y attache, dans le prolongement d'autres rencontres et publications collectives qui ont fait la part belle à l'exploitation des données LiDAR'

À l'origine de ce numéro spécial, il y a eu deux journées d'étude organisées par les auteurs de cet article. La première, le 22 novembre 2018, intitulée «Archéologie en sous-bois », eut lieu au centre

\footnotetext{
${ }^{1}$ Nous pensons ici notamment à la session «Archaeology in the Woods: New Technologies, New Perspectives » dans le cadre du CAA2014 (Giligny et al. 2015), au Workshop 2 du programme européen ERC Rurland «Parcellaires conservés sous forêt», 5 mai 2014 : https://rurland.hypotheses.org/category/publications-rurland-sur-hal/workshop-2-paris-5mai-2014 (lien valide le 4 juin 2020), à une précédente rencontre organisée à Paris $1:$ «Applications du Lidar en science géohistorique », 9-10 novembre 2016 : https://abp.hypotheses.org/files/2017/05/Affiche-Livret-LIDAR.pdf (lien valide le 4 juin 2020), au « Trail 2016 » du réseau Isa, intitulé Training and Research on the Archaeological Interpretation of LiDAR, 17-20 mai 2016, http://isa.univ-tours.fr/spip.php?article342 (lien valide le 4 juin 2020), pour ne citer que des événements organisés en France. 
Michelet de 1'université Paris 1 Panthéon-Sorbonne et rassembla une soixantaine de personnes. La seconde, le $1^{\text {er }}$ février 2019, intitulée «Applications du Lidar en archéologie », se tint à la Maison de l'archéologie et de l'ethnographie (MAE) à Nanterre, réunissant un auditoire d'une trentaine de personnes. Pour ces deux occasions, nous avions convié des personnalités d'horizon divers. Il s'agissait tout d'abord d'échanger entre chercheurs, enseignants-chercheurs ou doctorants de Paris 1 travaillant au sein de diverses UMR sur l'exploitation de données LiDAR en Bourgogne (Christophe Petit, Alain Giosa et Clément Membrivès), en Auvergne (Laure Laüt) et au centre du Mexique (Antoine Dorison et Grégory Pereira).

Nous souhaitions aussi élargir les débats en invitant des chercheurs d'autres universités ou laboratoires, dont les travaux portent aussi sur des espaces forestiers. C'est ainsi que nous avons eu le plaisir d'accueillir des spécialistes d'approches environnementales venus de Nancy (Jean-Luc Dupouey) ou de Besançon (Vincent Bichet) mais aussi des doctorants des universités de Limoges (Graziella Rassat) ou de Dijon (Rémi Landois) qui ont respectivement présenté des recherches portant sur l'Alsace, le Jura, les Charentes et la Bourgogne (fig. 1 et 2).

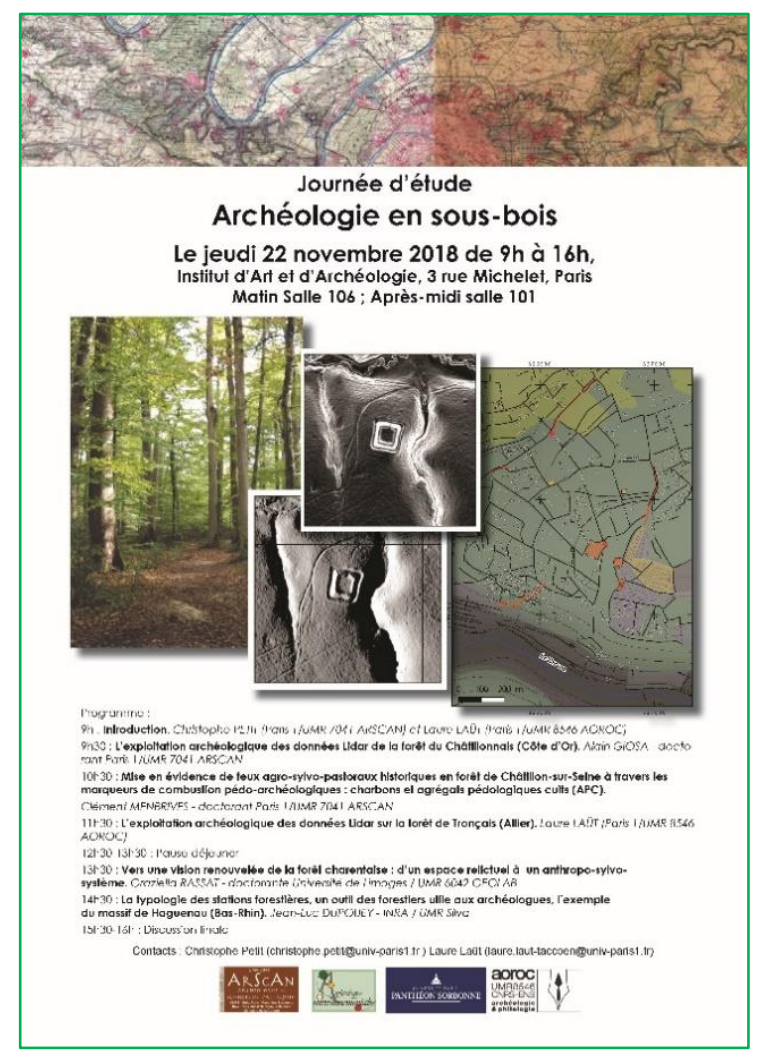

Fig. 1. Affiche de la journée d'étude du 22 novembre 2018

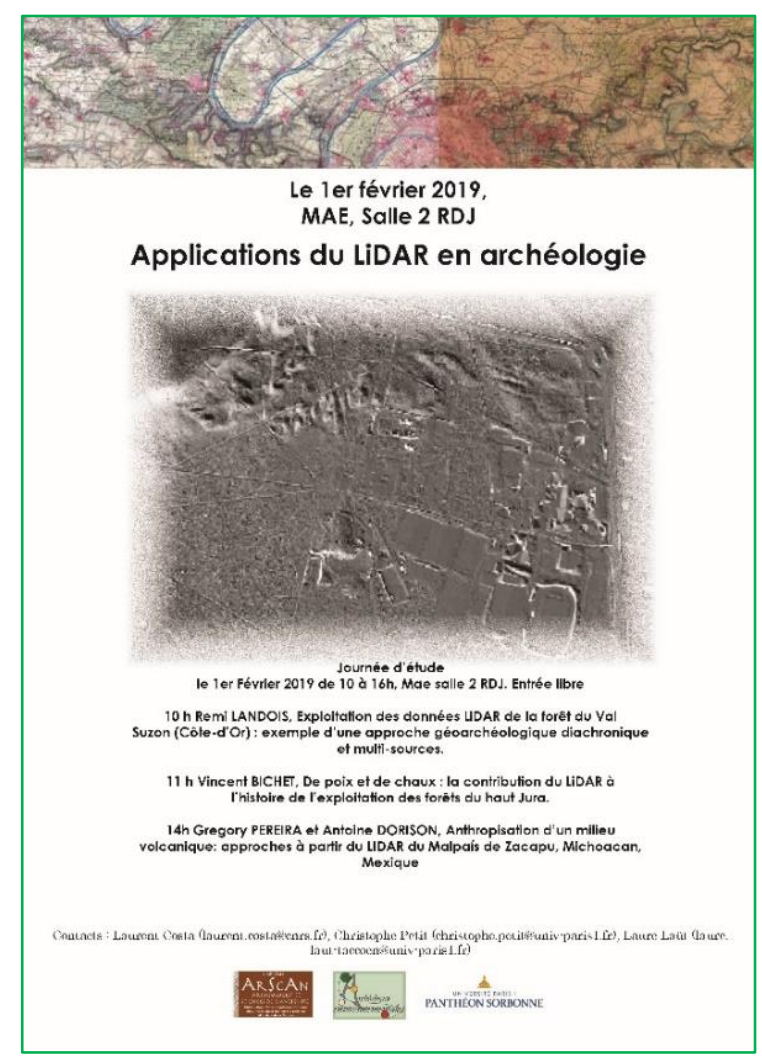

Fig. 2. Affiche de la journée d'étude du $1^{\text {er }}$ février 2019

Ces deux journées d'étude suscitèrent des discussions et des échanges fournis, faisant rapidement germer l'idée de passer de la parole aux «actes», en proposant aux intervenants la publication de leur communication dans un numéro spécial de la revue Archéologies numériques. Quatre auteurs ont ainsi accepté de rédiger un article, parfois avec le concours de collaborateurs, comme JeanPierre Garcia et Amélie Quiquerez sur la forêt de Val-Suzon. Certains orateurs de nos journées d'étude n'ont pas pu participer à ce projet, mais nous renvoyons ici à leurs travaux universitaires ou publications déjà disponibles sur les questions abordées, concernant les forêts de Charente (Rassat et al. 2015, 2018), du Châtillonnais (Giosa 2019, Membrivès 2016), du Jura (Bichet et al. 2019, Chevassu et al. 2017) ou de Haguenau (Dupouey 1983, Abert et al. 2010).

D'autres chercheurs ont été sollicités par la suite, pour étoffer le contenu de ce numéro spécial et nous avons été heureux de voir affluer les réponses positives, avec des propositions d'articles. C'est 
ainsi que sont venues s'ajouter des contributions de Pierre Fetet, d'Isabelle Le Tellier-Heitz, de Murielle Georges-Leroy, de Catherine Fruchart (en collaboration avec François Favory), de Jérôme Spiesser, de Rémi De Matos Machado, Jean-Pierre Toumazet et Stéphanie Jacquemot et enfin de Magali Watteaux et Olivier Nihesse. Ce sont donc au total 10 articles qui sont rassemblés dans ce numéro, sans compter cette introduction et la conclusion générale, dans laquelle nous avons brossé un récapitulatif des différentes structures étudiées dans ces différentes contributions. Que les 16 auteurs ou collaborateurs qui ont œuvré à la réalisation de ce projet soient vivement remerciés ici.

Cet enchaînement positif nous laisse mesurer l'attente, voire l'enthousiasme de la communauté scientifique vis-à-vis de ces questions. Nous vivons en effet un véritable tournant dans la recherche, grâce à la technologie LiDAR, qui permet un renversement de situation historique en archéologie. Dans les années 1970-80, le développement de la prospection aérienne a révélé d'innombrables structures insoupçonnées auparavant, en particulier dans les vastes zones d'openfield de l'aprèsremembrement (Delétang 1999, Nuninger 2018,). Puis se développa l'archéologie préventive, qui a accompagné les mouvements d'aménagement du territoire dans les années 1990-2000 (Demoule 2007). "The place to be» pour l'archéologue de terrain a donc longtemps été l'espace ouvert, les vastes étendues cultivées ou la terre en cours d'artificialisation des franges urbaines et des voies rapides. Mais aujourd'hui, la technologie LiDAR ouvre le champ de l'enquête archéologique sur tous les espaces forestiers, qui avaient été jusqu'à présent largement délaissés, voire volontairement fuis car supposés pauvres en vestiges. Et l'on y découvre aussi des richesses insoupçonnées qui, à leur tour, renouvellent assez largement notre vision des paysages, des dynamiques de peuplement et de la mise en valeur des terres. Revanche donc de ces forêts devenues pénétrables. Ces derniers développements viennent renforcer le travail mené depuis longtemps par les pionniers de l'archéologie forestière qui avaient, bien avant l'arrivée du LiDAR, compris le potentiel archéologique des forêts et commencé à mettre en place des protocoles d'approche adaptés, avec une attention particulière aux reliefs (Debordes 1973a et b, Ghestem, Vilks 1979, Lecquoy 1986, Lecomte 1997, Vieille 2001, Dardignac et al. 2003, Decocq et al. 2004, Dupouey et al. 2007). Signe des temps aussi, dans une période qui a vu le retour de la friche et de la forêt, avec les surfaces boisées qui ont doublé en France depuis le milieu du XIX ${ }^{\mathrm{e}}$ siècle (Koerner 2000), où l'on renonce à de grands projets d'aménagement comme EuropaCity, où l'on crée un label «forêt d'exception » et de nouveaux parcs nationaux, comme celui, tout récent, des forêts de Champagne et Bourgogne.

Ce numéro spécial nous offre l'occasion d'un tour d'horizon des approches françaises en matière d'exploitation archéologique du LiDAR sans prétendre, loin de là, à l'exhaustivité, tant les travaux sont foisonnants, en France comme à l'étranger. Mais l'éventail des articles ici réunis reflète la diversité des recherches actuelles, quant aux échelles de travail (site, massifs forestiers, région...), aux procédés d'acquisition LiDAR (avion, drone), aux terrains d'enquête (de l'Aisne à l'Allier, de la Vendée aux Vosges et jusqu'au Mexique) ou aux périodes et fourchettes chronologiques abordées (du temps long de l'histoire agraire au temps court de la bataille de Verdun). Ce sont aussi d'assez nombreux points de méthodes qui sont abordés au fil des articles, concernant le traitement des données LiDAR, l'interprétation manuelle ou semi-automatique des reliefs, les protocoles de vérification sur le terrain, la confrontation avec les données environnementales, etc.

Autant d'éclairages qui, nous l'espérons, viendront témoigner de cette recherche pleine de relief (à tous les sens du terme...) et nourrir la réflexion sur les apports du LiDAR à l'enquête archéologique en forêt, sur les approches à développer pour bien l'exploiter, en travaillant la donnée numérique, mais aussi et toujours l'information historique et la réalité du terrain.

\section{Bibliographie}

Beck, P. 2009. « Saint-Martin-du-Mont (Côte-d’Or). Bordes des Bois de Cestres ». Archéologie médiévale 39 (2009) : 201. 
Bessière, F. \& V. Guichard 2010. «Chronique des recherches sur le Mont Beuvray: 2006-2008». Revue archéologique de l'Est [En ligne] 59, $\mathrm{n}^{\mathrm{O}}$ 1-2010 (5 janvier 2012). ttp://journals.openedition.org/rae/6502.

Bichet, V., A. Barbier, V. Chevassu， D. Daval, E. Gauthier， M. Montandon, H. Richard \& M. Thivet 2019. «Traverser les montagnes du Jura: identification de voies antiques de franchissement de la haute chaîne jurassienne par analyse LiDAR. » dans : Des routes et des hommes : la construction des échanges par les itinéraires et les transports. 2019.

Canuto, M., A. F. Estrada-Belli, T. G. Garrison, S. D. Houston, M. Jane, J. Acuña, M. Kováč, D. Marken et al. 2018. «Ancient Lowland Maya Complexity as Revealed by Airborne Laser Scanning of Northern Guatemala ». Science 361, n 6409 (28 septembre 2018) : eaau0137. https://doi.org/10.1126/science.aau0137.

Dardignac, C., G. Bénailly \& T. Vigneau 2003. «Forêt et archéologie : inventorier, gérer et protéger le patrimoine de nos forêts ». Rendez-vous techniques $\mathrm{n}^{\mathrm{o}} 2$ (automne 2003).

David, S. \& F. Trouvé 2015. «Montmorency (Val-d'Oise) : une forêt passée au laser». Nouvelles de l'Archéologie, $\mathrm{n}^{\circ} 139$ (mars 2015): 45-50.

David, S. 2018. «Les nouvelles découvertes archéologiques en forêt de Fontainebleau : l'apport des données Lidar pour la connaissance d'un territoire ». La Voix de la Forêt, 2018, 81, à paraitre, 22-24.

Decocq G., B. Doyen \& P. Thuillier 2004. Archéologie des milieux boisés en Picardie, Revue archéologique de Picardie, vol 1, n 1, p. 149-164.

Delétang, H. (dir.) 1999. L’archéologie aérienne en France : le passé vu du ciel, Errance, Paris, 173 p.

Demoule, J.-P. (dir.) 2007. L'archéologie préventive dans le monde: apports de l'archéologie préventive à la connaissance du passé (Collection «Recherches »). Découverte, Paris, 286 p.

Desbordes, J.-M. 1973a. Principes d'archéologie forestière, Archéologia, 69 : 60-65.

Desbordes, J.-M. 1973 b. La recherche archéologique sous-bois, Revue archéologique de Picardie [En ligne], 3, 1 : 12.

Devereux, B. J., G. S. Amable \& P. Crow 2008. «Visualisation of LiDAR terrain models for archaeological feature detection. » Antiquity 82, n 316 (2008): 470-79.

Doneus, M. 2013. «Openness as Visualization Technique for Interpretative Mapping of Airborne Lidar Derived Digital Terrain Models ». Remote Sensing 5, $\mathrm{n}^{\mathrm{0}} 12$ (28 novembre 2013): 6427-42. https://doi.org/10.3390/rs5126427.

Dupouey, J.-L. 1983. «Étude phytosociologique et écologique du massif forestier de Haguenau (Bas-Rhin) - Apports méthodologiques - Potentialités sylvicoles ». Sciences de la Terre. AgroParisTech, 1983. Français. 〈tel-00589411〉

Dupouey, J. -L., E. Dambrine, C. Dardignac \& M. Georges-Leroy 2007. La mémoire des forêts. Actes du colloque «Forêt, archéologie et environnement»14-16 décembre 2004. [En ligne], Office national des forêts, Institut national de la recherche agronomique, Direction régionale des affaires culturelles de Lorraine, [s.1.], 294 p.

Forest, M., L. Costa, A. Combey, A. Dorison \& G. Pereira 2020. «Testing Web Mapping and Active Learning to Approach Lidar Data». Advances in Archaeological Practice 8, $\mathrm{n}^{\circ} 1$ (février 2020): 25-39. https://doi.org/10.1017/aap.2019.42.

Gallagher, J. M. \& R. L. Joseph 2008. «Using LiDAR to detect cultural resources in a forested environment: an example from Isle Royale National Park, Michigan, USA ». Archaeological Prospection 15, $\mathrm{n}^{\circ} 3$ (2008) : 187-206.

Georges-Leroy, M., J. Bock, E. Dambrine \& J.-L. Dupouey 2009. «Le massif forestier, objet pertinent pour la recherche archéologique. L'exemple du massif forestier de Haye (Meurthe-et-Moselle)», Revue Géographique de l'Est [En ligne], 49, vol. 49/2-3

Georges-Leroy, M., J. Bock, E. Dambrine \& J. L. Dupouey 2011. «Apport du lidar à la connaissance de l'histoire de l'occupation du sol en forêt de Haye », ArcheoSciences. Revue d'archéométrie [En ligne], 35 : 117-129.

Georges-Leroy, M., J. Bock, É. Dambrine \& J.-L. Dupouey 2013. «Apport du lidar à la connaissance de l'histoire de l'occupation du sol en forêt de Haye » ArcheoSciences.

Georges-Leroy, M., J. Bock, E. Dambrine, J.-L. Dupouey \& J.-D. Laffite 2014. « Parcellaires et habitat antiques des forêts du plateau de Haye en Lorraine : bilan et perspectives », in : Les parcellaires conservés sous forêt [En ligne], [s.n.], Paris, France.

Giligny, F., F. Djindjian, L. Costa, P. Moscati \& S. Robert (dir.) 2015. CAA2014. $21^{\text {st }}$ Century archaeology. Concepts, methods and tools. Proceedins of the $42^{\text {nd }}$ Annual Conference on Computer Applications and Quantitative methods in Archaeology, Archeopress, Oxford, 649 p. 
Ghestem, A. \& A. Vilks 1979. La végétation de quelques sites archéologiques en Limousin. Premières recherches sur la relation entre les plantes et les structures archéologiques, Revue archéologique du Centre de la France [En ligne], 18, $3: 137-148$.

Giosa, A. 2019. «Les agrosystèmes antiques du Châtillonnais. Approche archéopédologique de la mémoire des forêts dans les parcellaires reconnus par télédétection LiDAR ». Thèse de doctorat soutenue le 20 décembre 2019, Paris 1 Panthéon-Sorbonne.

Goguey, D., J. Bénard, M. Berranger, A. Cordier, G. Jobelin \& E. Vega (dir.) 2018. Structures en pierre du plateau du Châtillonnais (Côte-d'Or) : du Hallstatt à l'Antiquité tardive : l'apport de l'archéologie forestière, Archéologie et histoire romaine $\mathrm{n}^{\circ}$ 39, Éditions Mergoil, Dremil-Lafarge, 289 p.

Goguey, D., Y. Pautrat, J.-P. Guillaumet, J.-P. Thevenot \& L. Popovitch 2010. Dix ans d'archéologie forestière dans le Châtillonnais (Côte-d'Or) : enclos, habitats, parcellaires, Revue archéologique de l'Est [En ligne], Tome 59-1.

Hall, B., G. Motzkin, D. R. Foster, M. Syfert, \& J. Burk 2002. « Three Hundred Years of Forest and Land-Use Change in Massachusetts, USA ». Journal of Biogeography 29, n 10-11 (octobre 2002) : 1319-35.

Koerner, W., B. Cinotti, J. H. Jussy \& B. Marc 2000. «Évolution des surfaces boisées en France depuis le début du $\mathrm{XIX}^{\mathrm{e}}$ siècle : identification et localisation des boisements des territoires agricoles abandonnés » Revue Forestière Française, ${ }^{\circ} 3$ (2000) : 249. https://doi.org/10.4267/2042/5359.

Landois, R., J.-P. Garcia \& A. Quiquerez 2019. «Un habitat groupé antérieur au XII siècle préservé sous forêt : le site de Goa (Val-Suzon, Côte-d'Or)». Bulletin du centre d'études médiévales d'Auxerre, BUCEMA [En ligne] 23, $\mathrm{n}^{\circ} 1$ $\left(1^{\text {er }}\right.$ septembre 2019). https://doi.org/doi.org/10.4000/cem.16385.

Le Jeune, Y., C. Dardignac \& S. David 2017. «Bercé avant la forêt Premiers résultats de l'exploitation des cartes anciennes et des données lidar sur la forêt de Bercé (Sarthe)», Revue Forestière Française, LXIX, 4-5 : 519-543.

Lecomte, F. (dir.) 1997. «Archéologie et forêt », Arborescence, $71:$ 2-41.

Membrivès, C. 2016. Recherche d'indices d'activités agro-sylvo-pastorales liées à l'emploi du feu dans un terroir archéologique préservé sous forêt: approche pédo-anthracologique en Châtillonnais (Côte-d'Or), Mémoire de recherche en Master 2, 2016, Université Paris 1 Panthéon-Sorbonne.

Mestre, M., M. Hildebrand \& P. Texier 2008. «Un exemple d'utilisation du LiDAR sous couvert forestier guyanais ». Archéopages 23 (2008) : 69-75.

Nuninger, L. 2018. «Du ciel au territoire, vers une nouvelle perception de l'espace archéologique », in: Une histoire des civilisations. Comment l'archéologie bouleverse nos connaissances, La Découverte/INRAP., 569-73. Paris, 2018. https://hal.archives-ouvertes.fr/halshs-01420568 (lien valide le 8 juin 2020).

Rassat, G., F. Cerbelaud, R. Crouzevialle, J. -P. Toumazet, P. Allée, N. Dieudonné-Glad \& M.-C. Bal-Serin 2018. "L'utilisation et l'apport des données LiDAR pour l'étude des forêts en Charente », in : Paradis-Grenouillet S. (dir.), Charbonnage, charbonniers, charbonnières : confluence de regards autour d'un artisanat méconnu, Presses universitaires de Provence, Aix-en-Provence, p. 103-118.

Rassat, G., J. -P. Toumazet, F. Cerbelaud, R. Crouzevialle, P. Allée, N. Dieudonné-Glad \& M.-C. Bal 2015. «Le LiDAR: pour une histoire renouvelée des forêts charbonnées de plaine charentaises. Approche classique et détection semi-automatisée des plates-formes de charbonnage, poster, in : $\mathrm{XX}^{\mathrm{e}}$ colloque d'archéométrie du GMPCA [s.n.], [s.l.].

Rassat, S. \& L. Hugonnier 2017. «Atteindre 1'histoire de la forêt de Compiègne par la télédétection aérienne et l'exploration des archives du sol », Histoire \& Mesure, Vol. XXXII, 2 : 67-102.

Risbøl, O, A. K. Gjertsen, \& K. Skare 2006. "Airborne laser scanning of cultural remains in forests: some preliminary results from a Norwegian project", in : Proceedings of the $2^{\text {nd }}$ International Workshop (BAR International Series 1568), 107-12. CNR, Rome, Italie.

Saligny, L. \& M. Mémier 2014. “Expertise et suivi d'une acquisition Lidar pour l'archéologie : l'exemple d'Autun. Journée d'actualité archéologique en pays Eduen". Autun: Service archéologique de la Ville d'Autun. hal01153253.

Sittler, B. \& K. Hauger 2004. "Les apports du laser aéroporté à la documentation de parcellaires anciens fossilisés par la forêt : l'exemple des champs bombés de Rastatt en Pays de Bade", 155-61. ONF-INRA-DRAC Lorraine.

Vidal, J. 2016. "Mode d'occupation du site d'Alésia (Alise-Sainte-Reine, Côte-d'Or). Analyse spatiale multiscalaire des données archéologiques issues des fouilles, de l'imagerie géophysique et de la télédétection (photographie aérienne et LiDAR)". Thèse de doctorat, Université de Bourgogne-Franche-Comté. 
Vieille, V. 2001: “Analyses floristiques et phyto-sociologiques de la végétation de quelques mottes castrales picardes". Thèse Pharmacie, Amiens, 93 pages.

Weishampel, J., A. Chase, D. Chase \& S. Leisz 2012. «The Geospatial Revolution in Mesoamerican Archaeology ». Proceedings of the National Academy of Sciences 109 (32) (s. d.): 12916-21. 